\title{
Use of 3D models for control of the main pipeline protective facilities
}

\author{
Maria Shlyakhova ${ }^{1}$ \\ ${ }^{1}$ Siberian State University of Geosystems and Technologies, Photogrammetry and \\ Remote Sensing Department, 10 Plakhotnogo street, 630108, Novosibirsk, Russia
}

\begin{abstract}
The primary purpose of main pipelines is to transport oil products from a point to the point of destination. Therefore, its length can be thousands of kilometers, most often it is located in difficult natural and climatic conditions. Main pipeline continuous operation and protection from accidents or breakdowns requires passive or active control of protective structures using 3D technologies. The article briefly describes the technology for creating 3D models based on materials from aerial laser scanning and aerosurvey.
\end{abstract}

\section{Introduction}

Oil products are generally transported by pipelines associated with the risk of accidents and, in turn, an oil spill. The use of 3D models to control the main pipeline protective structures is an urgent task, for it allows obtaining realistic data on the state of the surveyed object and subsequent decision making on identifying any deviations from the specified parameters. The accuracy and detail of 3D models will mainly depend on the selected information collection technology. There is a classification of protective structures depending on the type of material.

Depending on the material of a main pipeline protective structure, two main types are distinguished: earthen and concrete. The authors [1] identified the main criteria for the accuracy control (Tab. 1).

Table 1. The choice of data collection technology depending on the type of protective structure.

\begin{tabular}{|c|c|c|c|}
\hline $\begin{array}{c}\text { Type of protective } \\
\text { structure }\end{array}$ & $\begin{array}{c}\text { Accuracy in } \\
\text { plane view }\end{array}$ & $\begin{array}{c}\text { Accuracy in } \\
\text { elevation }\end{array}$ & $\begin{array}{c}\text { Data collection technology for } \\
\text { creating 3D models of protective } \\
\text { structures }\end{array}$ \\
\hline Earthen structure & $15 \mathrm{~cm}$ & $15 \mathrm{~cm}$ & Aerosurvey, aerial laser photography \\
\hline Concrete structure & $10 \mathrm{~mm}$ & $15 \mathrm{~mm}$ & Aerial and ground laser scanning \\
\hline
\end{tabular}

In the northern regions, the main protective structures are earthworks, which can be reasonably controlled by methods of aerial laser scanning and aerosurvey. When using this technology for collecting information, the following types of data are obtained: a digital model of the relief and terrain, as well as information about the surface texture [2,3]. The following technological processes are used to build a 3D model (Fig. 1) [4]. 


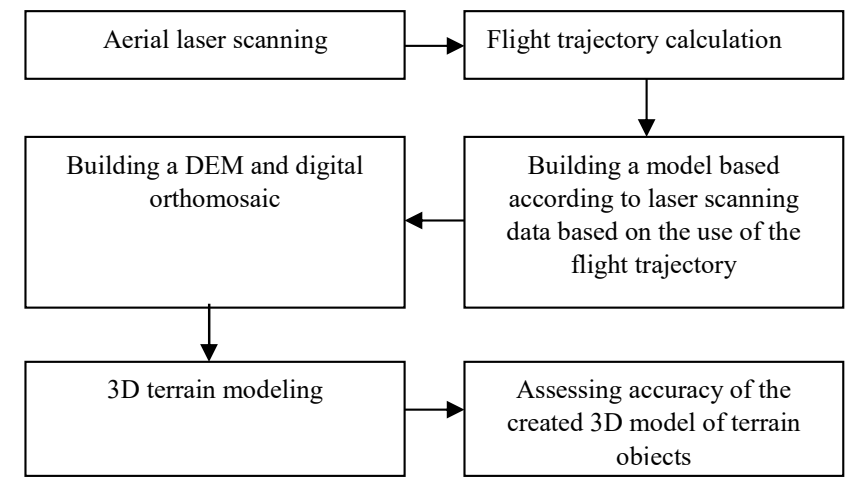

Fig. 1. Laser scanning data processing and digital aerial photography scheme for creating 3D models

\section{Experiment}

Stereophotogrammetric and laser surveys were performed using a Leica ALS-60 laser scanner and a Leica RCD 105 digital aerial camera to create 3D models of main pipelines and protective structures (Fig. 1). The surveys were accompanied by measurements of onboard GPS and IMU. Terra Solid software product based on the Bentley MicroStation platform was used to process the data (Figure 1).

The images were used to measure the plane coordinates of the object image points and to calculate spatial coordinates of the object points $(\mathrm{X}, \mathrm{Y}, \mathrm{Z})$, which are the points of the spatial terrain model. An image element belongs to each point of the object. Brightness of an element and an image for a terrain point (a pixel in a terrain) can be taken from any image belonging to a set of overlapping images in the corresponding point area. Then the coordinates of the points were recalculated into the screen plane coordinate system $\mathrm{x}, \mathrm{y}, \mathrm{z}$, and brightness of the element with $\mathrm{x}, \mathrm{y}$ coordinates was assigned previously defined brightness of the point on the terrain model with $\mathrm{X}, \mathrm{Y}, \mathrm{Z}$ coordinates.

Figure 2 shows the airborne laser scanning data processed in the Terra Solid software. 


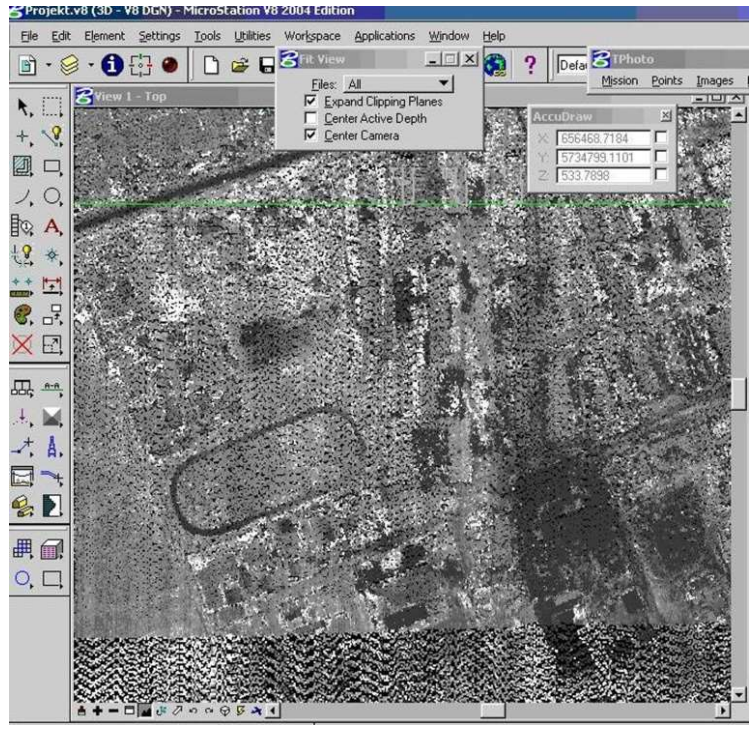

Fig. 2 - Airborne laser scanning processing data in PP Terra Solid

The next stage was building a digital elevation model and digital orthomosaic. As a result, a 3D hedgehog was created, and the accuracy was assessed by determining the points on the photographic image and the finished model, after which the affine transformation coefficients and the texture element coordinates were found. The result of evaluating the root-mean-square error accuracy is $0.33 \mathrm{~m}$ in elevation and $0.57 \mathrm{~m}$ in plane view. [5]

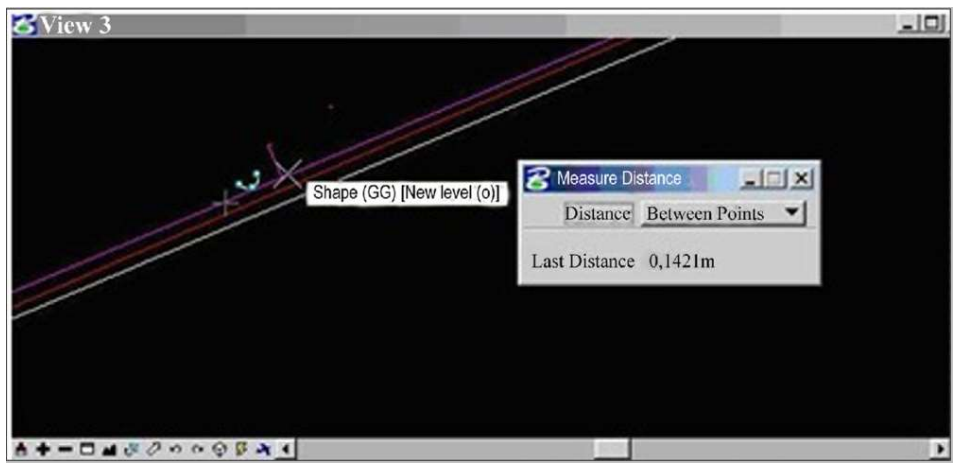

Fig. 3. Measuring the discrepancy between models.

To build a realistic 3D measurement model, it is necessary to perform a large number of measurements of characteristic points, which must also be identified in the images from which the image texture is taken. In addition to coordinate transformations, a real texture of the image should be formed when building 3D models.

In most modern technologies, the texture is oriented relative to the object surface by identifying image points on the texture and in a 3D mode. Then affine transformation 
coefficients are determined and coordinates of the texture elements are recalculated into the corresponding elements of the 3D model. Thus, the texture only approximately corresponds to the object and is not a measurement one. In addition, standard aerial survey (with $40 \%$ and $60 \%$ overlap) is not sufficient for texture generation, so additional images are required to create a realistic model.

Thus, knowing the accuracy of the protective structure control and the choice of survey methods, we can create 3D models with maximum accuracy in order to timely identify potentially dangerous sections of the pipelines and reduce the risk of accidents.

The information obtained can be used to create $3 \mathrm{D}$ models that will help to timely identify emergencies, and control reliability of the pipeline by monitoring it throughout the entire period of operation. 3D models also make it possible to track changes and automatically simulate situations in the three-dimensional form of the deformation area.

Thus, the use of 3D models helps:

- Make automatic measurements of geometric parameters of communication;

- Exercise control over the raw material transportation and extraction;

- Update information based on terrain change data;

- Monitor the state of communications;

- Simulate emergency situations based on the various sensors readings (pressure, temperature) and prevent them.

It should be noted that raw materials can be transported in various ways, but the pipeline is still the most profitable and safe way. In order to avoid emergency situations, it is necessary to carry out $3 \mathrm{D}$ monitoring using remote sensing methods, and to build $3 \mathrm{D}$ models with maximum accuracy and detail based on the information obtained. With the help of such models, problems are identified and analyzed at pipeline sections in order to quickly eliminate accidents or breakdowns. Therefore, environmental disasters can be avoided.

The work was carried out within the framework of the state assignment of the Ministry of Education and Science of Russia (subject: Development of theory and technological solutions for monitoring the state of protective structures when pumping oil products using active remote sensing methods, No. 0807-2020-0002).

\section{References}

1. A.V. Komissarov, M.M. Shlyakhova, M.A. Altyntsev, E.N. Kulik. Criteria for the main pipeline protective structure control. SSUGT Bulletin. 2020. V. 25, No. 4, pp. 96-104.

2. V.V. Dedkova, M.M. Shlyakhova. Monitoring of the main pipeline technical condition of using remote sensing methods. Regional problems of the Earth remote sensing of: proceedings of the VII International Scientific Conference, Krasnoyarsk, 29 Sept. - 2 Oct. 2020, pp. 192-195.

3. M.M. Shlyakhova, V.V. Dedkova. Prospects for the use of aerial surveys for the control of main pipeline protective structures. Regional problems of the Earth remote sensing of: proceedings of the VII International Scientific Conference, Krasnoyarsk, 29 Sept. 2 Oct. 2020, pp. 316-319.

4. A.P. Guk, M.M. Shlyakhova. Development of methods for creating 3D models from aerospace images of high and ultra-high resolution and other remote sensing data. Izvestiya VUZov. Geodesy and aerial photography. 2011. No. 2, pp. 32-34.

5. A.P. Guk, M.M. Shlyakhova. Some problems of building realistic 3D measurement models from remote sensing data. SSUGT Bulletin. 2015. V. 2, No. 4, pp. 51-60.

6. A.P. Guk, M.M. Shlyakhova, R.V. Brezhnev. Potential of using realistic 3D models built from digital images to solve a wide range of problems. National scientific and 
practical conference Regulation of Land and Property Relations in Russia, 2018. SSUGT. V. 1, pp. 172-176. 